\title{
EXPERIMENTAL INVESTIGATION OF THE NATURAL DIVERTOR CONFIGURATION IN HELIOTRON-E*
}

D.L. Hillis, P.K. Mioduszewski, R.H. Fowler, and J.A. Rome

Oak Ridge National Laboratory, Oak Ridge, Tennessee 37831, USA

O. Motojima, T. Mizuuchi, N. Noda, T. Mutoh, H. Zushi, R. Takahashi,

T. Obiki, A. liyoshi, and K. Uo

Plasma Physics Laboratory, Kyoto University, Uji, Japan

Keywords: heliotron, helical divertor, particle control

CONF $-880512--4$

DE88 010444

\section{Abstract}

Particle control with pump limiters and divertors has been successfully demonstrated in a number of present-day tokamaks. In a heliotron/stellarator configuration, plasma flows to the wall in distinct flux bundles, often called "diverior stripes". This complicated three-dimensional characteristic of the plasma edge presents a new challenge for active particle control systems such as pump limiters and divertors. The experiment described here has obtained data with an ; strumented pumped particle collector that is located in the "natural" magnetic divertor stripe of Heliotron-E. The particle collector consists of a moveable graphite assembly with single-sided particle colleciion and active pumping.

By scanning the particle collector assembly through the plasma edge of Heliotron$\mathrm{E}$, the divertor stripe is observed to be about $2-3 \mathrm{~cm}$ (FWHM) in width, and pressure rises of 0.1-0.2 mTorr are observed in the particle collector pumping chamber.

These measurements have demonstrated that particles leaving the buik plasma via the divertor stripes can be collected and provide a basis for developing a diverter scheme for particie control in helical systems. Modeling of the Heliotron-E magnetic configuration at the plasma edge is used to determine the collection efficiency of the particle collector in the divertor stripes. The modeling is further extended to describe a helical divertor concept.

'Research sponsored by the Office of Fusion Energy, U.S. Department of Energy, under contract DE-AC05-840R21400 with Martin Marietta Energy Systems, Inc.

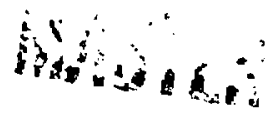




\section{Introduction}

The control of particle flux, heat flux, and impurity generation at the vacuum vessel wall is crucial to the realization of a fusion reactor. Stellarator/heliotron [1-3] devices offer an attractive alternative to tokamaks as fusion reactors because of their prospects for steady-state, high-beta operation without plasma current. As these helical systems achieve long-pulse and steady-state operation, it will be necessary to provide plasma density and impurity removal schemes to optimize plasma performance. Recent experiments in tokamaks have produced encouraging results for density (particle) control and impurity control using pumped limiters [4-6] and magnetic divertors $[7,8]$. For the heliotron/stellarator configuration, work has only just begun to develop particle and impurity removal schemes. In ref. [g] it was demonstrated that density control could be achieved in Heliotron-E with a conventional tokamak- type pump limiter, if the limiter was placed inside the last closed magnetic flux surface. This configuration, with a material limiter in contact with the plasma core, inherently introduces additional impurities and does not take advantage of the natural magnetic separatrix of the heliotron corffiguization. Recent experiments with divertor tokamaks have produced excellent results $[7,8]$ but require additional complex coil systems. For a heliotron-type device the separatrix layer of the helical magnetic field produces a "natural" divertor action without additionai coils[10,11]. The main goal of these Heliotron-E experiments is to obtain experimental data on particle flow and edge plasma properties with an instrumented pumped particle collector, which is located in the "natural" magnetic divertor stripe of Heliotron-E, arid thereby assess the possibility of particle and impurity control using the natural divertor action of a heliotron/stellarator configuration.

\section{Experimental details}

\section{DISCLAIMER}

2.1. The Heliotron-E device

This report was prepared as an account of work sponsored by an agency of the United States Government. Neither the United States Government nor any agency thereof, nor any of their employees, makes any warranty, express or implied, or assumes any legal liability or responsibility for the accuracy, completeriess, or usefulness of any information, apparatus, product, or process disclosed, or represents that its use would not infringe privately owned rights. Reference herein to any specific commercial product, process, or service by trade name, trademark. rnanufacturer, or otherwise does not necessarily constitute or imply its endorsement, recommendation, or favoring by the United States Government or any agency thereof. The views and opinions of authors expressed hercin do not necessarily state or reflect those of the United States Government or any agency thereof. 
The Heliotron-E device is a helical toroidal system that has an $\ell=2$, $m=19$ helical field with large rotational transform angle $t$ and shear [3]. The major radius of the device is $2.2 \mathrm{~m}$, and the magnetic surfaces are elliptical as shown in fig. 1. The average minor radius of the plasma is about $0.2 \mathrm{~m}$. Currentless hydrogen target plasmas are produced at a toroidal field of $B=1.9 \mathrm{~T}$ with electron cyclotron heating $(\mathrm{ECH})$ using $300 \mathrm{~kW}$ of $53.2-\mathrm{GHz}$ power. Near the end of the $50 \mathrm{~ms}$ ECH pulse, up to $3.4 \mathrm{MW}$ of neutral hydrogen is injected into the $\mathrm{ECH}$ target plasma for $150 \mathrm{~ms}$. For these experiments, typical plasma parameters are $\overline{n_{e}} \approx 3 x$ $10^{13} \mathrm{~cm}^{-3}, T_{e} \approx 800 \mathrm{eV}$ and $T_{i} \approx 700 \mathrm{eV}$ for $3.2 \mathrm{MW}$ of neutral beam injection(NBI).

\subsection{Magrietic configuration}

The last closed magnetic flux surface of Heliotron-E (determined by the separatrix region of the helical magnetic field) is shown in fig. 1. The hatched region shows the poloidal cross section of the Heliotron-E divertor region [12], along with indications of localized divertor traces on the vacuum vessel wall. Field lines in the hatched region pass near the separatrix and emerge in well-defined helical stripes near the vessel wall. The divertor stripes rotate poloidally by $360^{\circ}$ as they progress through one helical field period. The modeling of the magnetic configuration and divertor stripes is calculated using a multifilament model of the helical coil currents, as described in ref. [13]. From these calculations, the connection length of the field line between the plasma and the wall for the hatched region is $>5 \mathrm{~m}$. For even larger radii, $r>31.5 \mathrm{~cm}$ (inside the hatched region), the connection length is very short $(\leq 1 \mathrm{~m})$ and the field lines are even more strongly diverted to the wall.

Previous experiments on Heliotron-D [5] and on Helir,tron-E [12] indicate that about $90 \%$ of the charged particles diffuse from the well-confined plasma volume into the divertor layer and flow to the wall along the divertor stripes. To model particles flowing along the divertor stripes to the wall, field lines were launched randomly $1-2 \mathrm{~cm}$ outside the last closed flux surface and followed until they hit the wall. The three-dimensional view in fig. 2 shows the results of this calculation for one 
field period of Heliotron-E. Two helical divertor stripes clearly occur on the outside wall of Heliotron-E. The coordinate $Y$ is the distance above and below the horizontal midplane, $Z$ is the distance measured toroidally along one helical field period, and $R$ is the distance measured along the major radius.

\subsection{The particle collector}

To obtain experimental data on particle flow and edge plasma properties within the natural divertor stripes of Heliotron- $E$ a moveable particle collector module was installed on a horizontal midplarie port, as shown in fig. 1. The collector assembly has a stroke of $58 \mathrm{~cm}$ and permits the positioning of the particle collector near the vacuum vessel wall or up to $6 \mathrm{~cm}$ inside the last closed flux surface. This range of positions permits the study of a plasma limited by the magnetic separatrix or a heliotron plasma limited by a material limiter. The particle collector assembly has a graphite head with single-sided particle collection; the remainder of the structure is stainless steel.

The size of the particle collector head is restricted to $\approx 14.7 \mathrm{~cm}$ in diameter by the size of the poit holes on Heliotron-E. To begin the design of a particle collector head that is matched to the particle flow along the divertor stripes the magnetic iield line calculations shown in fig. 2 are used. The large circle in fig. 2 represents a $14.7 \mathrm{~cm}$ diameter collector head and indicates that a sizeable number of field lines (or particles) would intercept the particle collector head. Additional magnetic field line calculations were performed; the total number and location of the field line intersections with the collector head are recorded as a function of the position of the collector head with respect to the last closed flux surface in fig. 3 . The black dots are the intersections of the field lines with the collector head, and the values of $x$ represent the distance in centimeters from the collector head to the last closed flux surface. At $x=0$ the tip of the particle collector head is just tangent to the last ciused flux surface, and at $x=-2 \mathrm{~cm}$ the particle collector tip is $2 \mathrm{~cm}$ outside the last closed flux surface. The two divertor stripes clearly move across the collector head as the position of the head is moved from $-2 \mathrm{~cm}$ to $-5 \mathrm{~cm}$ outside the last closed flux surface, 
with a maximum clustering of the field lines around $x=-4 \mathrm{~cm}$. These calculations indicate that the ar:gle of incidence of the field lines with the collector head is about $75^{\circ}$ as measured with respect to a normal (taken along the major radius) to the tip of the collector head.

The entrance window for the particle collector is chosen to best match these calculations at $x=-4 \mathrm{~cm}$ (see figs. 2 and 3 ). The collector siot is $2.1 \mathrm{~cm}$ high (along the major radius), $1.9 \mathrm{~cm}$ wide, and $7 \mathrm{~cm}$ long, with the length extending along the top face of the graphite head (see inset of fig. 1). Calculations were also performed by assuming that particles with an energy of $1 \mathrm{keV}$ were flowing out along the divertor stripe to see if this changed the divertor stripe locations or widths in figs. 2 and 3. In general, the divertor stripes occurred at the same location on the divertor head but tended to be broadened by about $20-30 \%$. From these modeling calculations, $=0.6 \%$ of the field lines that were started raridomly $1-2 \mathrm{~cm}$ outside the last closed flux surface should end up in the particle collector slot when it is positioned at $x \approx-4 \mathrm{~cm}$.

Active pumping for the system is provided by a Zr/Al getter pump [6], which is located directly behind the collector head. The nominal pumping speed for thermal hydrogen is measured to be $\approx 1000 \mathrm{~L} / \mathrm{s}$ for the particle collector configuration. The pressure in the particle collector chamber is measured with a Schulz-Phelps gauge. When the $\mathrm{Zr} / \mathrm{Al}$ pump is not activated and pressure equilibrium is attained, the measured pressure corresponds to the actual pressure in the particle collector chamber. When the pump is activated a pressure gradient along the getter cartridge must be accounted for, and the measured pressure must be multiplied by a factor of 3 to give the pressure near the entrance slot of the particle collector.

The locations of the graphite head and particle coliection window relative to the last closed flux surface of Heliotron-E are shown in fig. 1 . Measurements of the plasma electron temperature and density in the scrape-off layer are provided by a double Langmuir probe located just inside the slot of the particle collector, while the total energy scraped off by the graphite particle collector head is measured by an embedded thermocouple. The particle flux to the particle collector is deduced by 
using a fast Schulz-Phelps ionization gauge (10 $\mathrm{ms}$ response time) to measure the pressure rise in the particle collector chamber. The Schulz-Phelps gauge is located at the end of an extension tube with a vacuum time constant of about $20 \mathrm{~ms}$. To observe hydrogen recycling from the graphite head when it is located in the divertor stripe region, a collimated photodiode with a bandpass filter views the $\mathrm{H}_{\alpha}$ emission from the vicinity of the particla collector head.

\section{Experimental Results}

To investigate the particle flow and edge plasma properties within the divertor stripes, the particle collector position, $x$, is varied from the vacuum vessel wall to $6 \mathrm{~cm}$ inside the last closed flux surface both before and after activating the particle collector getter pump. Figure 4 shows the pressure rise observed in the particle collector as a function of the collector position for a series of identicai 1.0-and 3.2MW NBI discharges for $\bar{n}_{e}=3 \times 10^{13} \mathrm{~cm}^{-3}$ with the pumps "off". Outside the last closed flux surface $(x<0)$, the pressure buildup is observed to be a maximum of 0.12 mTorr at $x=-4 \mathrm{~cm}$, which is where the divertor stripe is predicted to illuminate the particle collector slot. The measurement also indicates that the width of the divertor stripe is about $2-3 \mathrm{~cm}$ FWHM (full width at half maximum). For $x \geq 0$, the HeliotronE paricle collector behaves very much like a conventional tokamak pumped limiter [9] and the pressure in ureases dramatically as the collector is inserted farther into the well-confined plasma.

To investigate the differences in plasma parameters on Heliotron- $E$ as the plasma is limited by the magnetic separatrix or by the particle collector, the plasma parameters $T_{e}, T_{j}, \bar{n}_{e}$, and the radiated power $P_{\text {rad }}$ are measured as the particle collector position is varied. The quantity $P_{\text {rad }}$ is the radiation loss of the plasma as measured by a calibrated bolometer. These plasma parameters are unaffected for $x<2 \mathrm{~cm}$; however, for $x>2 \mathrm{~cm}, T_{e}, T_{j}$, and $\bar{n}_{e}$ begin to fall sharply and the radiated power increases (see fig. 3 of ref. [9]) dramatically. The increase in radiated power is accompanied by an increase in carbon and iron impurities generated from the particle collector head. This indicates that the particle collector head should reside 
outside the last closed flux surface, where it does not affect the plasma parameters.

The relationship between the plasma density in the divertor stripe, $n_{e, d}$, and the pressure in the particle collector is investigated with a double Langmuir probe located at the entrance to the particle collector slot. Figure 5 shows the electron density $n_{e, d}$ in the divertor stripe and the $H_{\alpha}$ emission from the collector head as a function of the particle collector position for a series of identical 3.2-MW NBI discharges at $\bar{n}_{e}=3 \times 10^{13} \mathrm{~cm}^{-3}$ with the pumps "off". As was seen in fig. 4 for the pressure rise, $n_{e, d}$ and the $H_{\alpha}$ intensity both reach a maximum at the expected location, $x=-4 \mathrm{~cm}$. The electron temperature $T_{e, d}$ in the divertor stripe, as measured by the double Langmuir probe, is found to be $\approx 15 \mathrm{eV}$. The increase in $H_{\alpha}$ also indicates an increase in the hydrogen recycling near $x=-4 \mathrm{~cm}$.

When active pumping of the collector was provided by the $\mathrm{Zr} / \mathrm{Al}$ getter pump, the pressure in the collector chamber was decreased by about a factor of 8 from that in the unpumped case at $x=-4 \mathrm{~cm}$. During this active pumping phase the core plasma density $\bar{n}_{e}$ was measured; and no changes in $\bar{n}_{e}$ were observed as a result of active pumping of the module. Of course, with such a small collector slot and such low densities in the divertor stripes, significant density (particle) control should not be expected. The power to the particle collector head at $x=-4 \mathrm{~cm}$ was about $6 \mathrm{~kW}$, as measured by the embedded thermocouple in the graphite collector head.

\section{Discussion of Results}

A large pressure buildup (1-10 mTorr) and high edge densities ( $>5 \times 10^{12}$ $\mathrm{cm}^{-3}$ ) [4] to efficiently remove particles from the plasma. To prevent impurity generation and gas recycling from the particle collector head, it is desirable to locate the particle collector outside the last closed flux surface in the separatrix or divertor region of Heliotron-E. In the divertor region, impurities and gas recycling from the particle collector would be somewhat insulated from the plasma core. If the particle collector headi is placed inside the last closed magnetic flux surface at $x=0$, then the 
plasma boundary is defined not by the magnetic separatrix, but by the graphite particle collector head. In the previous Heliotron-E pumped limiter measurements [9] with similar operating conditions, pressures of $=1 \mathrm{mTorr}$ were observed for a similar unpumped configuration at $x=3 \mathrm{~cm}$, while the pressure in the present particle collector at $x=3 \mathrm{~cm}$ is only $\approx 0.2 \mathrm{mTorr}$. The present particle collector window is very small and is improperly oriented, as compared to the pump limiter of ref. [9], thereby reducing its collection efficiency mark $\in$ dly for $x \geq 0$.

For particle collector positions outside the last closed flux surface $(x \leq 0)$, pressures of only 0.05-0.1 mTo:r are observed, with correspondingly small densities in the divertor stripes $\left(<5 \times 10^{11} \mathrm{~cm}^{-3}\right)$. For the small particle collector module used here, the edge density in the divertor stripes is too low and the area of the cuiliector slot $\left(\approx 13 \mathrm{~cm}^{2}\right)$ too small to collect a large fraction of the particles flowing out the divertor stripes in each of the 19 helical field periods. The total surface area of the divertor stripes on the vessel wall is estimated to be $\approx 8 \%$ of the total surface area of the vessel wall [11]. Direct evidence of this divertor action is seen as localized erosion [12] on the wall, where these divertor traces are expected. Clearly, to make major changes in the plasma density a large fraction of the divertor stripe area must be sampled.

The data of figs. 4 and 5 are best discussed by considering the particle flux into the limiter. Using the values of $n_{e, d}$ and $T_{e, d}$ measured for the divertor stripe, the particle flux into the collector slot can be estimated as $\Gamma_{\|}=n_{e, d} v_{\|}$, where $v_{\|}$ $\approx 0.3 C_{S}$ and $C_{S}$ is the ion sound speed. This yields a particle flux to the limiter of $\Gamma_{H}$ $\approx 9 \times 10^{17} \mathrm{~cm}^{-2} \mathrm{~s}^{-1}$, which is small compared to conventional tokamak scrape-off layers, where $\Gamma_{\|} \approx\left(10^{19}-10^{20}\right) \mathrm{cm}^{-2} \mathrm{~s}^{-1}[14]$. This agrees with the estimates derived from the Schulz-Phelps ion gauge for the neutal flux of molecules in the limiter chamber, $\Gamma_{0}$, which can be calculated according to Eq. (6) of ref. [15]. To determine $\Gamma_{0}$, the parameters $\lambda$ (decay length of the particle flux) and $\eta$ (the collection probability) must be known, in addition to the measured pressure $p$ in the collector and the height $h$ of the pumping slot. From the observed neutral pressure of $\approx 0.12 \mathrm{mTorr}$ in the particle collector chamber along with $\lambda=1.5-4 \mathrm{~cm}[16], \mathrm{h}=2$ $\mathrm{cm}$, and $\eta \approx 0.7$ [15], the incident flux $\Gamma_{0} \approx(7-10) \times 10^{17} \mathrm{~cm}^{-2} \mathrm{~s}^{-1}$. Therefore, 
both flux measurements indicate that the particle flux within the divertor stripe ( $x=-4$ $\mathrm{cm})$ is quite low $\left(=9 \times 10^{17} \mathrm{~cm}^{-2} \mathrm{~s}^{-1}\right)$.

The total plasma efflux $\phi$ of particles for Heliotron-E is $\phi=\bar{n}_{e} V_{p} / \tau_{p}$. where $\bar{n}_{e} \approx 5 \times 10^{13} \mathrm{~cm}^{-3}, V_{p}$ is the plasma volume, and $\tau_{p}$ is the global particle confinement time. The value of $\tau_{p}$ was recently measured for Heliotron-E [17] by Balmer alpha laser fluorescence spectroscopy and $\tau_{p}$ is $\approx 10-20 \mathrm{~ms}$. For these values $\phi$ is about $2.4 \times 10^{21} \mathrm{~s}^{-1}$. From the modeling of the magnetic geometry of Heliotron-E, about $0.6 \%$ of the field lines that were started $1-2 \mathrm{~cm}$ outside the last closed flux surface should enter the slot of the particle collector head. If we assume that about $90 \%$ of the particles that leave the well-confined plasma diffuse into the divertor layer, flow out along the divertor traces, and either hit the wall or enter the collector slot, then we find that about $2 \times 10^{19}$ particles /s should enter the particle collector slot. From the neutral pressure and Langmuir probe measurements, the plasma flux into the particie collector is $\Gamma_{\|}=9 \times 10^{17} \mathrm{~cm}^{-2} \mathrm{~s}^{-1}$; combining this value with the area of the collector slot yields $\approx 1.2 \times 10^{19}$ particles $/ \mathrm{s}$ entering the collector slot. The theoretical modeling of the divertor stripes and the measurements tend to agree quite well as to the magnitude of particles (or field lines) that should enter the particle collector slot.

To efficiently collect particles in the divertor stripes, a large fraction of the surface area of the helical divertor stripe must be covered. This can be accomplished for Heliotron- $E$, as shown in fig. 6 , by providing a helically slotted liner that permits the diverted plasma to flow into a tokamak-like divertor chamber and strike a graphite target plate, where the heat, particle, and impurity flux can be removed. The helical divertor configuration produced by using the "natural" divertor action provided by the helical magnetic field is termed the "Natural" Helical Divertor (NHD). Obiki et al. [18] have modeled this configuration for Heliotron-E, and a 2field-period slotted helical divertor chamber is being fabricated to determine the particle collection capabilities of such a hybrid divertor system. This 2-field-period prototype helical divertor system will be tested on Heliotron-E during the summer of 1988 and should intercept about $10 \%$ of the total divertor stripe area of Heliotron-E, thereby collecting a large fraction of the plasma efflux. The pressure buildup and 
exhaust efficiency in such a helical divertor system depend critically on the design, orientation, and dimensions of the helically slotted divertor chamber. These experiments should further enhance the understanding of particle removal schemes for the new longer-pulse helical systems that are just beginning operation, such as the Advanced Toroidal Facility (ATF) and Wendelstein VII-AS, and for the next generation of Heliotron-type devices.

\section{Summary}

The collection of particles from the naturally occurring divertor stripes of Heliotron-E has been successfully demonstrated with a graphite particle collector module that was placed well outside the last closed magnetic flux surface and within the $h \epsilon^{\prime \prime} \cap$ al divertor stripes. The width of the divertor stripes for Heliotron-E was measured to be about $2.5 \mathrm{~cm}$ (FWHM), and the location of the stripes agrees well with theoretical calculations that model the location of the divertor stripes. The theoretical calculations also provided a basis for determining the number of particles that could be expected to enter the particle collector slot. These predictions agreed quite well with the experimental measurements. Sinc $\theta$ the particle flux in the divertor stripes is observed to be quite low, $\approx 9 \times 10^{17} \mathrm{~cm}^{-2} \mathrm{~s}^{-1}$, it is necessary to collect a large fraction of the surface area of the helical divertor stripe. To accomplish this, a helical divertor chamber is proposed, which would take advantage of the natural divertor action provided by the helical magnet coils to form a Natural Helical Divertor (NHD). 


\section{References}

[1] K. Uo et al. , in: Heating in Toroidal Plasmas, Proc. 3rd Joint VarennaGrenoble Int. Symp., Grenoble, 1982, Vol. 2 (CEC, Brussels,1982), p. 667.

[2] K. Uo et al., in: Controlied Fusion and Plasma Physics, Proc. 10th European Conf., Moscow, 1981 (European Physical Society ,1981) E-1.

[3] K. Uo et al., in: Plasma Physics and Controlled Nuclear Fusion Research 1982, Proc. 9th Int. Conf., Baltimore, 1982, Vol. 2 (IAEA, Vienna, 1983) p. 209.

[4] P. Mioduszewski, J. Nucl. Mater. 111 \& 112 (1982) 253.

[5] D. M. Goebel, Fusion Technol. 10 (1986) 761.

[ö] K.H. Dippel et al., in: Plasma Physics and Controlled Nuclear Fusion Research 1984, Proc. 10th Int. Cont., London, U.K., 1984, Vol. 1 (IAEA, Vienna,1985) p. 249.

[7] M. A. Mahdavi et al. J. Nucl. Mater. 111 \& 112 (1982) 355.

[8] F. Wagner et al., in: Physics of Plasma-Wall Interactions in Controlled Fusion, Eds. D.E. Post and R. Behrisch (Plenum, New York, 1986) p. 931.

[9] D.L. Hillis et al., J. Nucl. Mater. 145-147 (1987) 496.

[10] K. Uo et al., J. Phys. Soc. Japan 16 (1961) 1380. 
12

[11] T. Mizuuchi et al., J. Nucl. Mater. 121 (1984) 3.

[12] O. Motojima et al., J. Nucl. N'ater. $128 \& 129$ (1984) 524.

[13] R.H. Fowler et al., Oak Ridge National Laboratory Report ORNLTM6293 (1978); D.K. Lee, Comput. Phys. Commun. 25 (1982) 181.

[14] P. Mioduszewski et al., J. Nucl. Mater. 121 (1984) 285.

[15] P. Mioduszewski, J. Nucl. Mater. 145 - 147 (1987) 798.

[16] O. Motojima et al., Nucl. Fusion 15 (1975) 985.

[17] D. Okano et al., J. Nucl. Mater. $145-147$ (1987) 504.

[18] T. Obiki et al. , Kyoto University, private communication (1987). 


\section{Figure Captions}

Fig. 1 Poloidal cross section of Heliotron-E showing the last closed magnetic flux surface and the magnetic divertor region (hatched region). The moveable graphite particle coilector head is detailed in the inset.

Fig. 2 Theoretical calculation of the magnetic field lines in the divertor region that are in the vicinity of the slotted particle collector head (shown in Fig. 1 and here represented by the large circle in the center of the figure). The small circles represent magnetic field lines that continue outward and strike the vacuum vessel wall of Heliotron-E.

Fig. 3. Theoretical calculation of the magnetic field lines that are launched randomly $2 \mathrm{~cm}$ outside the last closed flux surface and followed until they strike tive particle collector head shown in the inset of Fig. 1. The particle collector head is placed outside the last closed magnetic flux surface at various locations. At $x=-4 \mathrm{~cm}$, the collector is located $4 \mathrm{~cm}$ outside the last closed flux surface.

Fig. 4. Measured pressure in the particle collector module versus coilector position for 1.0 and $3.2 \mathrm{MW}$ of $\mathrm{NBI}$ at $\overline{\mathrm{n}}_{e}=3 \times 10^{13} \mathrm{~cm}^{-3}$ before activation of the getter pumps. Collector positions are measured relative to the last closed magnetic flux surface $(x=0)$; negative values of $x$ refer to positions outside the last closed flux surface.

Fig. 5. Electron density, $\mathrm{n}_{e, d}$, in the divertor stripe and $\mathrm{H}_{\alpha}$ emission measured from the vicinity of the collector head as a function of the particle collector position for $3.2 \mathrm{MW}$ of $\mathrm{NBI}$ at $\overline{\mathrm{n}}_{e}=3 \times 10^{13} \mathrm{~cm}^{-3}$ before activation of the getter pumps. The electron density is measured with a Langmuir probe located in 
the slot of the particle collector head.

Fig. 6. A possible active particle control system for a helical system, like Heliotron-E. The "natural" magnetic divertor action of Heliotron-E is shown directing the divertor stripes into a particle collection (divertor) chamber that is actively pumped and lirsd with graphite tiles for handling the heat and particle flux. Three poloidal cross sections of Heliotron-E are shown for one-half of a helical field period. 
HELIOTRON-E

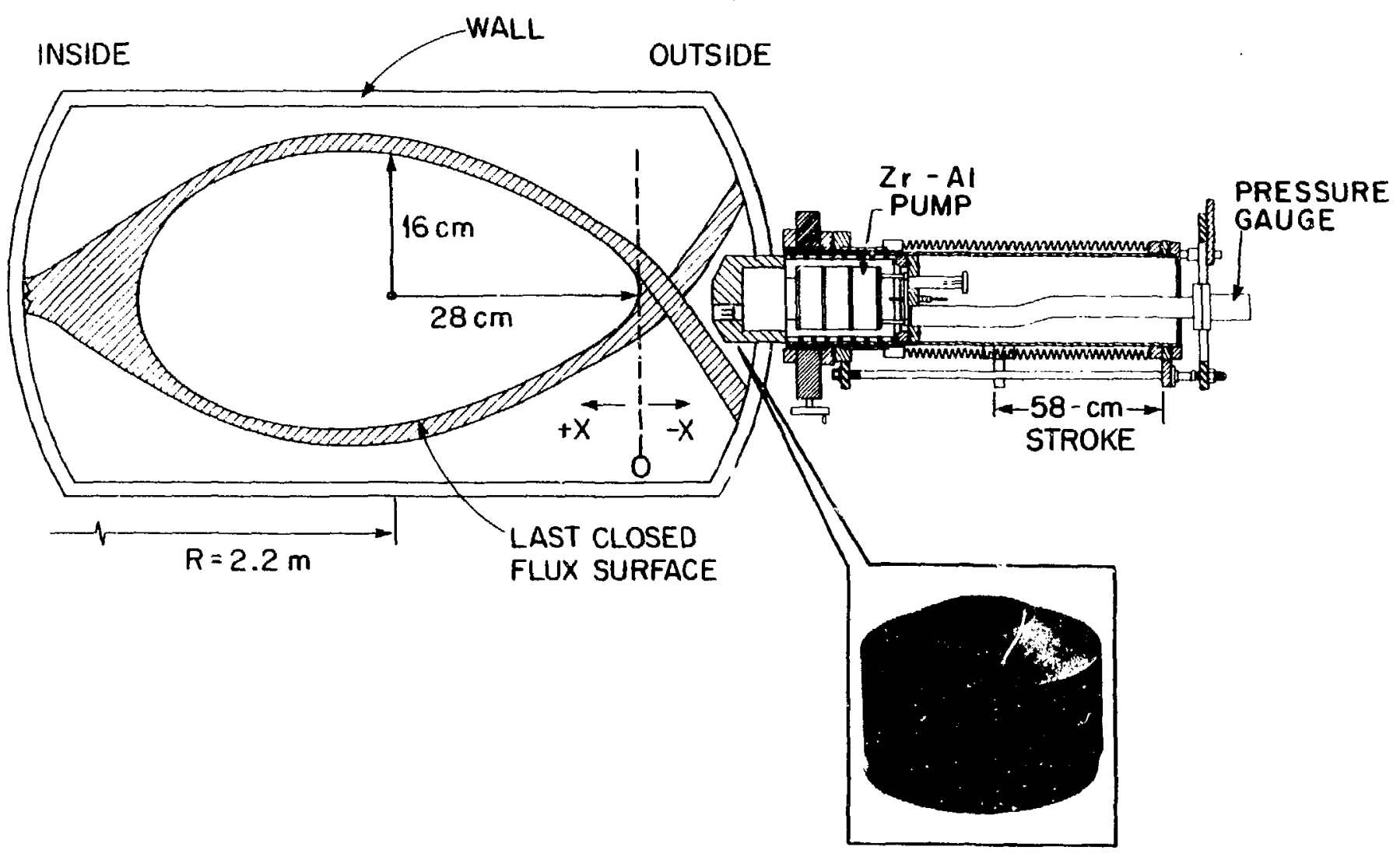




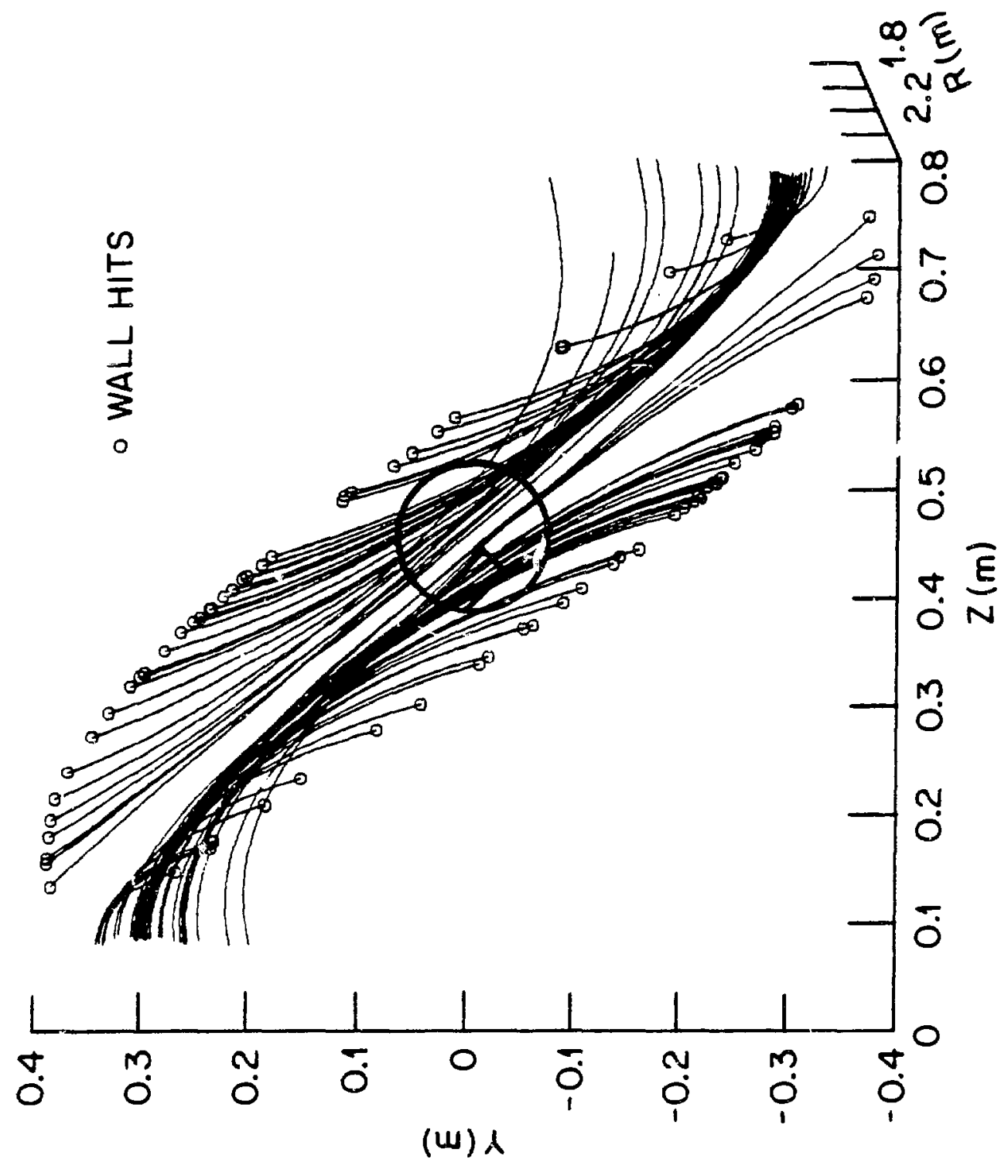

$\infty$ 

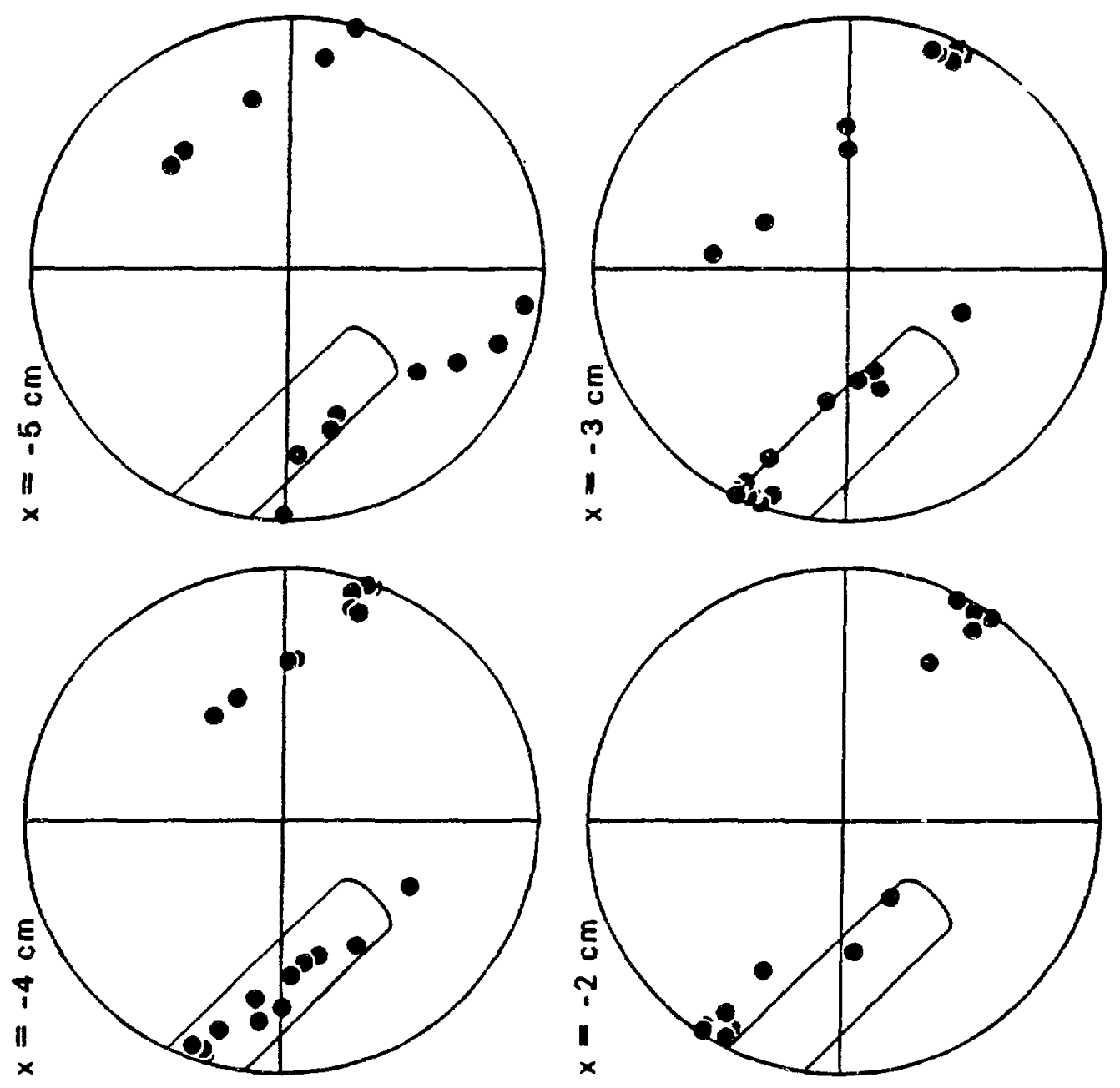

$i_{n}^{m}$ 


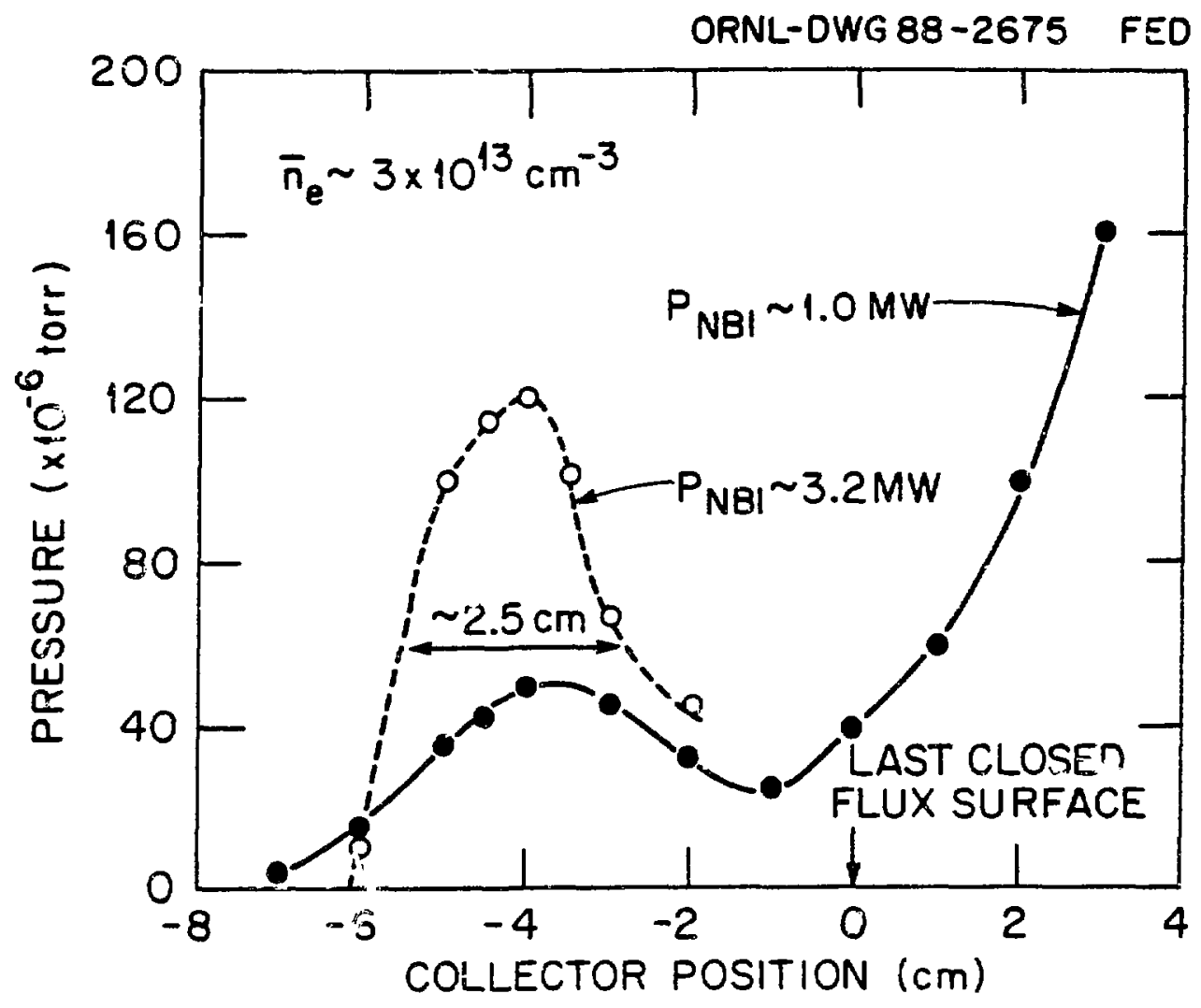

Fig. 4 


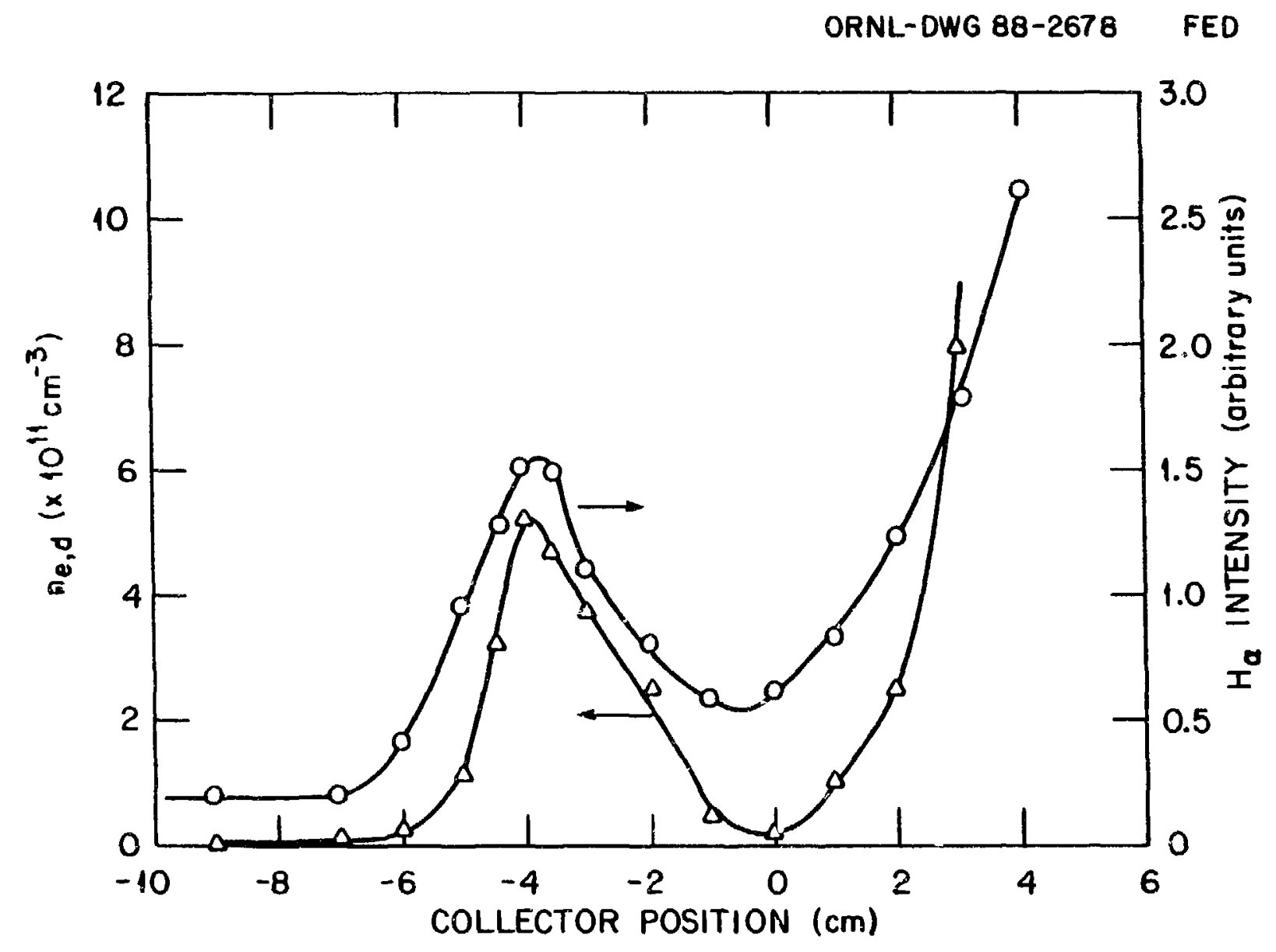

Fig. 5 


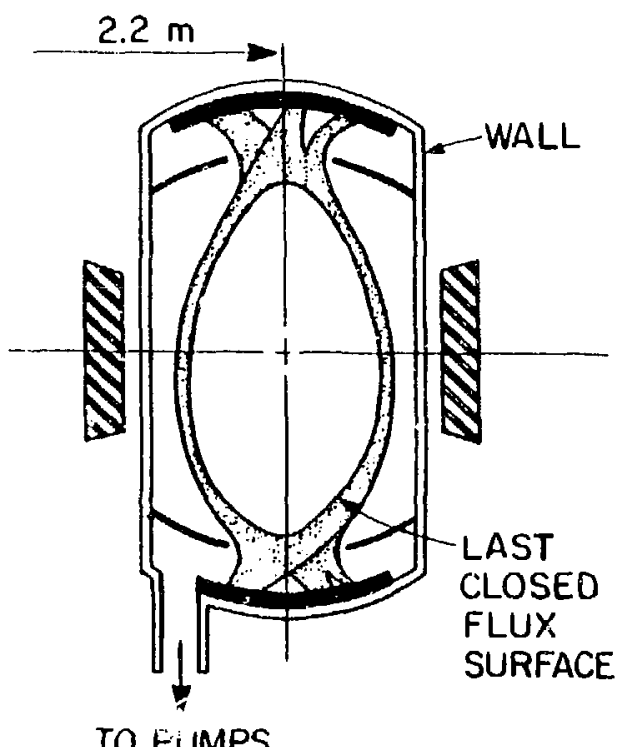

$\phi=0^{\circ}$

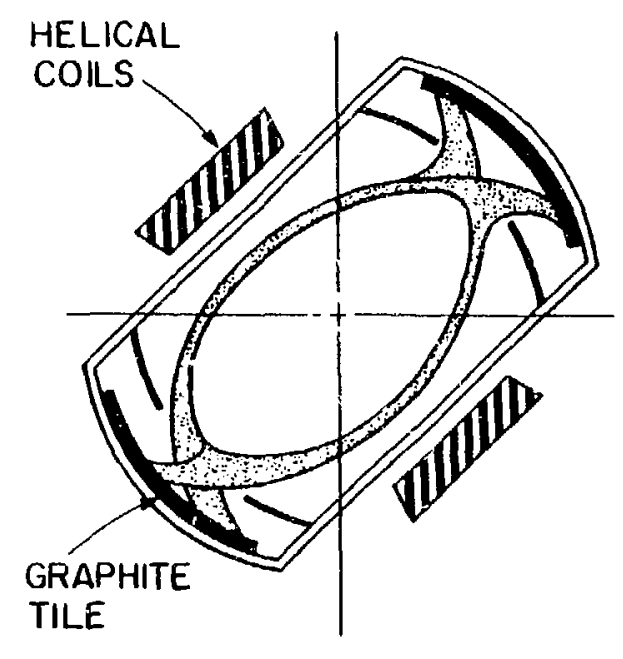

$\phi=4.75^{\circ}$

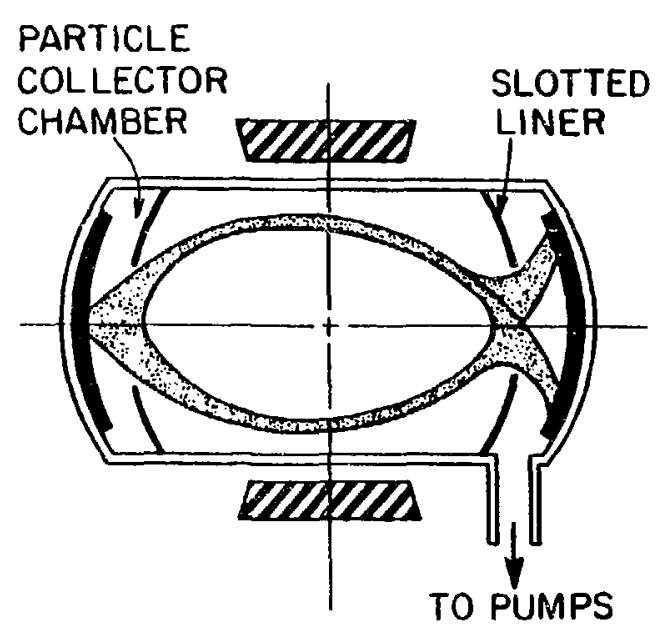

$\phi=9.5^{\circ}$ 